Théologiques

Théologiques

Posthumain, où es-tu ?

La condition humaine entre manque et totalité

Farid El Moujabber

Volume 19, numéro 2, 2011

URI : https://id.erudit.org/iderudit/1024734ar

DOI : https://doi.org/10.7202/1024734ar

Aller au sommaire du numéro

\section{Éditeur(s)}

Faculté de théologie et de sciences des religions, Université de Montréal

\section{ISSN}

1188-7109 (imprimé)

1492-1413 (numérique)

Découvrir la revue

\section{Citer cet article}

El Moujabber, F. (2011). Posthumain, où es-tu ? La condition humaine entre manque et totalité. Théologiques, 19(2), 189-207.

https://doi.org/10.7202/1024734ar
Résumé de l'article

Le " manque " et la « totalité » sont deux traits caractéristiques de la condition humaine dans sa quête de soi depuis les mythes fondateurs jusqu'au progrès phénoménal de la technologie au $\mathrm{XxI}^{\mathrm{e}}$ siècle. Néanmoins, ces deux concepts revêtent des connotations différentes et souvent contradictoires selon le contexte et les aspirations qui les sous-tendent. En quête de l'humanité parfaite, le posthumanisme prône la totalité comme le but ultime de la vie humaine, et refuse catégoriquement le manque dans son aspect négatif et écrasant. Par contre, une lecture sémiotique du texte de la Genèse révèle que le manque fait partie intégrante de la condition de l'homme, en opposition à la totalité qui s'avère mortelle. À cet égard, comment une nouvelle interprétation d'un texte datant de milliers d'années a le potentiel de jeter un regard nouveau sur des questions d'actualité ? Comment se déploient les espaces de la condition humaine entre manque et totalité ? 


\title{
Posthumain, où es-tu ?
}

\section{La condition humaine entre manque et totalité}

\author{
Farid El Moujabber* \\ Théologie et sciences religieuses \\ Université Laval (Québec, Canada)
}

Devant la maladie et la souffrance, l'être humain se trouve en état de manque $^{1}$ tant quant à la santé qu'au bien-être. Devant la mort, il réalise drastiquement son plus grand manque existentiel: l'immortalité. Cette problématique du manque qui conditionne l'être humain et la quête d'une totalité de son être ont marqué l'humanité depuis toujours. Dans l'Antiquité, des mythes ont évoqué une certaine plante de vie qui procure l'immortalité aux humains; dans le récit de la Genèse, Adam et Ève ont voulu être "comme des dieux»; des alchimistes du Moyen Âge ont été à la recherche de la jeunesse éternelle et de l'Élixir de vie; et de nos jours, la technologie promet à l'homme une victoire imminente sur la faiblesse et la mort.

Afin de vaincre le manque qui hante la condition humaine, la technologie met à la portée des humains des moyens inédits pour satisfaire des désirs enfouis dans le tréfonds de leur existence, surtout dans le domaine de la médecine et de la biologie. En envisageant le manque de la condition humaine à partir de son seul aspect biologique, la technologie ne fait qu'explorer des pistes de victoire technologiques dans le but de combler le manque et réaliser la totalité de l'être. Parmi ceux qui tirent profit des avancées de la technologie, les posthumanistes se présentent comme étant

* Farid El Moujabber est docteur en philosophie des sciences et stagiaire postdoctoral à la Faculté de théologie et sciences religieuses de l'Université Laval. Sa thèse s'intitule (2012) La nature humaine à l'ère de la technique moderne. Quelles mutations?. Il a récemment publié (2014) «Le posthumanisme: un épiphénomène moderne du dualisme», dans la revue Métabasis.

1. Le terme «manque " sera utilisé ici dans un sens non technique, mais plutôt d'ordre philosophique et général.

(C) Revue Théologiques 2011. Tout droit réservé. 
les techno-prophètes ${ }^{2}$ du siècle contemporain qui œuvrent pour le salut de l'humanité en transformant sa condition biologique en une condition postbiologique ${ }^{3}$ immortelle. En se servant de la médecine régénératrice, des nanotechnologies, de l'intelligence artificielle, etc., les posthumanistes cherchent à créer une nouvelle forme d'humanité exempte du manque.

En plus de leurs arguments scientifiques, les posthumanistes cherchent à appuyer leur quête de l'immortalité par des arguments théologiques tirés d'une lecture propre du texte de la Genèse. Ils considèrent que la condition de totalité posthumaine recherchée par le biais de la technologie pourrait représenter un retour à la condition de perfection initiale de l'homme, sans manque, dans le jardin d'Éden. Appliquer la technique sur l'homme en transformant son corps biologique n'est autre qu'une manière de se délivrer des conséquences mortelles de la «chute» du premier homme et de tout ce qui en découle comme manque. Cependant, une lecture sémiotique ${ }^{4}$ du texte de la Genèse révèle, à l'encontre de la lecture des posthumanistes, que le manque est caractéristique de la condition humaine dès l'origine, et qu'il est le moteur de toute créativité et de l'accomplissement de l'être humain. Dans ce contexte, la totalité, qui est censée être un idéal à atteindre, semble être mortelle pour l'homme.

Partant du postulat selon lequel «la vérité d'un texte est dans la capacité qu'il a d'être relu, et de donner à dire, dans son ancienneté, la nou-

2. À ce sujet, Dominique Lecourt dit: «C'est ce rêve même dont ceux que j'appelle les “techno-prophètes" s'enflamment encore aujourd'hui aux États-Unis. Leur foi dans la technologie est une véritable foi. Ils annoncent non la fin du monde ou la fin de l'humanité, mais l'entrée triomphale de notre espèce dans l'ère de la "posthumanité" grâce à l'intelligence artificielle» (Lecourt 2003, 44).

3. Ray Kurzweil, un des posthumanistes éminents du continent américain, postule que «the human species, along with the computational technology it created, will be able to offer succour to human needs and desires, and will be in a position to change the nature of mortality in a postbiological future» (Kurzweil 1999, 2).

4. La sémiotique fait partie des approches modernes de lecture des textes bibliques. En s'inscrivant dans le modèle de l'exégèse synchronique qui s'intéresse au texte tel qu'il se présente au lecteur dans son unité, elle se démarque de l'exégèse diachronique ou historico-critique. Jean-Marie Beaude explique que "la sémiotique s'intéresse, dans les textes, à la façon dont le sens se fait. Elle aborde le sens “à partir de ses conditions internes de production" [...] La sémiotique apparaît, de nos jours, comme un effort [vraiment] scientifique pour dire comment fonctionne le sens dans le discours oral ou écrit. Elle descend à un niveau de profondeur qui lui permet de repérer une organisation qui n'apparaît pas en surface du texte et pourtant l'organise » (Beaude 1979, 126-127). Cette interprétation a une portée philosophique et anthropologique qui va aider à mettre en relief la portée des choix philosophiques du posthumanisme. 
veauté d'un présent» (Panier 1996, 78), nous tenterons dans cet article de relire la condition posthumaine à la lumière d'une lecture sémiotique de la condition humaine telle que présentée dans le texte de la Genèse. En se basant sur les concepts de "manque " et de "totalité » qui constituent l'enjeu principal de la quête de perfection chez les posthumanistes et pour Adam et Ève, nous montrerons comment une nouvelle interprétation d'un texte datant de milliers d'années a le potentiel de jeter un regard nouveau sur des questions d'actualité. Ceci n'est possible que dans la mesure où l'enjeu du manque et de la totalité qui semble être théologico-scientifique dans la quête posthumaniste, et théologique dans le texte de la Genèse, s'avère être plutôt existentiel et anthropologique.

Dans un premier temps, nous commencerons par souligner le manque ressenti par l'être humain face à la performance de la machine et son aspiration à façonner un post-humain jouissant d'une condition postbiologique immortelle, et ce, en se référant aux arguments scientifiques de quelques grands penseurs posthumanistes, tels que Hans Moravec, Ray Kurzweil, Franc Tipler et Nick Bostrom. Nous puiserons par la suite dans les fondements théologiques et millénaristes de l'approche posthumaniste, afin de mettre au clair le lien entre le refus de la condition mortelle de l'homme et le désir de retrouver un état de perfection initiale inspirée d'une lecture posthumaniste du texte de la Genèse. Dans un deuxième temps, notre analyse portera sur une lecture sémiotique du texte de la Genèse selon l'exégète Paul Beauchamp, les théologiens Louis Panier et Jean Calloud, dans le but de présenter le manque, et non la perfection, comme faisant partie intégrante de la condition initiale de l'homme. À cet effet, deux aspects du manque seront déployés: l'aspect négatif du manque vécu dans un état de privation et poussant continuellement l'être humain à s'enfermer sur soi dans sa quête de la totalité; et un autre aspect plutôt positif qui a pour rôle d'enraciner l'homme dans ce qu'il est, et de l'ouvrir à une totalité qui se réalise avec autrui. Pour terminer, nous analyserons comment l' «espace» de la totalité pourrait être un espace de mort lorsque le «voir» et le «savoir» scientifique viennent remplacer l'«entendre» dans la relation de l'homme à Dieu et dans sa relation à autrui et à lui-même.

\section{Combler le manque de la condition humaine par la technologie}

À notre époque, le refus du manque au cœur de la condition humaine connaît une résurgence phénoménale avec le progrès de la technologie. En considérant le manque comme étant un défaut de la nature, chaque 
branche de la science cherche, dans son champ propre, à vaincre un certain aspect du manque qui frappe la condition humaine. À cet égard, les exemples se multiplient: les biotechnologies, les nanotechnologies, la médecine anti-âge, la médecine régénératrice, etc. Jean-Michel Besnier décrit ce refus dans une sorte de sentiment de honte et d'une «fatigue d'être soi » face à la performance des machines inventées par l'homme. Dans son ouvrage Demain, les posthumains, il dit:

Honteux d'être ce qu'ils sont - des «êtres seulement devenus ", victimes du fait d'être nés, soumis aux maladies et voués à la mort —, ils rêvent de mobiliser leur savoir aux fins d'y remédier: en se faisant être, ainsi que le permettraient le clonage et l'ectogenèse; en éradiquant les maladies, grâce à une médecine réparatrice des erreurs de la nature; et en s'assurant l'immortalité, au moyen de techniques sophistiquées au nombre desquelles figure en général celle du téléchargement de la conscience sur quelque support indestructible. (Besnier 2009, 138)

Ainsi, conscient de sa finitude, l'homme ressent la honte de détenir une condition humaine faible et mortelle, et cherche à surmonter cette faiblesse par l'entremise de la technologie se présentant à lui comme étant la solution miracle à l'angoisse d'être ce qu'il est. À cet effet, le posthumanisme, un courant scientifique originaire du continent américain, se donne pour mission de combler ce manque en se servant des prouesses de la technologie. La convergence des différentes technologies comme la nanotechnologie, la biotechnologie, l'informatique et la science cognitive l'appuie dans son projet faisant en sorte que la possibilité de vaincre la mort n'est plus invraisemblable, du moins théoriquement. Considérant la condition humaine actuelle comme étant désuète et problématique pour le progrès de l'humanité, les posthumanistes optent pour le remplacement du corps biologique mortel par un corps-machine robuste et durable. Si le corps biologique est le grand obstacle à l'immortalité de l'être humain, le corps postbiologique serait la solution technique à cette impasse, ouvrant ainsi l'humain à l'éternité. La perfection recherchée est ainsi matérialisée par une immortalité physique de l'être humain.

\subsection{Le projet posthumaniste de l'homme nouveau}

Afin de réaliser leur projet futuriste, les posthumanistes s'attaquent à la prémisse suivante: «La nature humaine est, et devrait rester essentiellement inaltérable" (Bostrom 2000, 1). Pour eux, il n'y a pas une soi-disant 
nature humaine ${ }^{5}$ fixe et inchangeable, car celle-ci confirmerait l'humanité dans son manque et condamnerait l'être humain à une condition humaine faible et imparfaite. Au contraire, le propre de l'homme, ce serait la transformation continuelle vers un meilleur avenir. En récusant les limites de la mortalité, les posthumanistes utilisent la technologie pour faire passer l'humanité à une condition posthumaine immortelle exempte de tout manque.

Pour ce faire, les posthumanistes se caractérisent par leur idéologie dualiste séparant le corps de l'esprit. Ce qui compte pour eux, c'est l'esprit ou l'intelligence de l'homme, non pas le corps dégradable. S'attacher au corps biologique signifie rester toujours assujetti à ses contraintes et continuer à souffrir de sa pesanteur. C'est la raison pour laquelle les posthumanistes dévalorisent la matière vivante du corps humain, source de manque et de faiblesse, et cherchent à la remplacer par une machine durable. De la sorte, le manque au cœur de la condition humaine serait vaincu par une simple intervention biotechnologique réglant ainsi l'angoisse existentielle de toute l'humanité depuis la nuit des temps.

Dans un premier temps, les posthumanistes cherchent à opérer une symbiose entre l'homme et la machine en commençant par équiper le corps humain de différentes extensions artificielles, pour ensuite œuvrer à télécharger l'intelligence humaine sur des machines entières, et plus tard créer des machines autonomes ayant la capacité de penser d'elles-mêmes et de se détacher de l'autorité des humains: "Such machines could carry on our cultural evolution, including their own construction and increasingly rapid self-improvement, without us, and without the genes that built us» (Moravec 1988, 2). Ce serait comme une nouvelle espèce plus évoluée qui dérive de notre espèce obsolète, mais qui la dépasse de loin, car ces machines auraient un jour une capacité très élaborée dans la combinaison des informations, et seraient par la suite capables de "penser».

Lorsque ces machines deviendraient pensantes, elles pourraient donc «exister» si nous prenons à la lettre le cogito cartésien «Je pense, donc je suis ». Pour illustrer ces propos, Frank Tipler, un physicien relativiste, montre dans son livre The Physics of Immortality que l'intelligence n'est que la capacité d'avoir un discours cohérent et logique. Subséquemment, si une machine peut avoir cette attitude et réagit positivement au test de

5. Les posthumanistes préfèrent à cet égard utiliser le concept de condition humaine à la place de "nature humaine ", parce qu'il prête à plus de plasticité, leur permettant de se lancer dans une transformation interminable du corps de l'être humain. 
Turing, elle peut être considérée comme intelligente et par la suite une personne: "The essential idea of the Turing test is that what counts for personhood is behavior: if it behaves in all respects like a person, then it is a person" (Tipler 1994, 21). Pour lui, sous l'angle physique, la "vie" est une forme de procession d'informations codées et préservées par la sélection naturelle, et l'«esprit » n'est autre qu'un programme d'ordinateur très complexe.

Frank Tipler fait remarquer que la définition traditionnelle de la vie se base sur le substrat "atome de carbone» comme fondement de la vie. Cependant, d'après Cairns $S \mathrm{mith}^{6}$, les ancêtres ultimes de la vie sont repérés dans un modèle d'autoreproduction qui se produisait à l'intérieur des particules de cristal métallique. Avec le temps, ce modèle a persisté, mais transféré dans un autre substrat: les molécules de carbone (voir Tipler 1994, 125). Ce qui importe donc, c'est le modèle et non le substrat, le modèle n'étant autre que l'ensemble des informations. Pourquoi alors refuser de substituer un substrat mécanique plus robuste à un substrat de carbone précaire? Si le corps fonctionne d'une manière mécanique, c'est qu'il ne constitue pas l'essentiel de l'être humain, et qu'il peut être facilement imité par n'importe quelle autre machine. Dans la lignée de la pensée cybernétique, remplacer le corps biologique par des machines performantes ne serait donc pour les posthumanistes que vaincre le manque et transformer le désordre en ordre, et l'éphémère en éternel.

Toute cette quête posthumaniste met en exergue chez eux le désir de combler le manque de la condition humaine, précisément la mort. Dans ce contexte, la mort n'est pas considérée comme une fin naturelle inscrite dans la condition humaine, mais comme une erreur de la nature. Corriger cette erreur consisterait à combler le manque qui frappe la condition humaine, et ainsi réaliser la vraie condition de l'homme qui, aux yeux des posthumanistes, devrait être une condition post-biologique immortelle.

Afin de fonder leur quête d'une perfection perdue exempte de la mort, les posthumanistes s'apparentent à une longue tradition millénariste fondée sur les visions apocalyptiques de l'Ancien Testament, et surtout sur l'Apocalypse de saint Jean dans le Nouveau Testament. La thèse milléna-

6. Graham Cairns Smith est chimiste et biologiste moléculaire à l'université de Glasgow. Il est devenu célèbre à cause de son ouvrage Seven Clues to the Origin of Life: A Scientific Detective Story où il défend sa théorie sur l'origine de la vie selon laquelle il conçoit une vie primitive minérale fondée sur un transfert d'information entre cristaux. 
riste de l'imperfection humaine vient renforcer leurs propos, et les confirme dans leur choix technique visant le perfectionnement de l'être humain.

\subsection{La quête d'une perfection perdue}

Dans le livre de l'Apocalypse, saint Jean annonce la deuxième venue du Christ pour un règne de mille ans (Ap 20,1-7) dans lequel Il va rétablir l'Éden perdu à la suite de la chute d'Adam, en le délivrant de tous les maux et précisément de la mort (1Co 15,26). Les millénaristes et le courant posthumaniste qui s'en inspire proposent une lecture fondamentaliste de ces textes bibliques. En effet, pour les millénaristes, le livre de la Révélation de saint Jean est un livre d'espoir qui vient annoncer la victoire de l'homme par un renversement du sort de la "chute». L'homme est appelé à retourner au paradis et à manger de l'arbre de vie afin de restaurer sa perfection perdue: "Il (Dieu) essuiera toute larme de leurs yeux, et la mort ne sera plus, et il n'y aura plus ni deuil, ni cri, ni douleur, car les premières choses ont disparu. Et celui qui était assis sur le trône dit: Voici, je fais toutes choses nouvelles» (Ap 21,4-5).

La première communauté chrétienne a cru que la réalisation de la victoire finale de l'homme était imminente, et saint Paul l'attendait de son vivant. Cependant, le christianisme officiel, surtout avec Origène et saint Augustin, a abandonné la croyance linéaire de la venue du Christ pour s'accrocher à sa venue eschatologique. Néanmoins, la croyance millénariste a persisté dans des communautés chrétiennes sous différentes formes selon les époques et les cultures, s'exprimant souvent dans la sphère scientifique par le culte de la science et de la technologie:

If millenarian expectations inspired the opening up of the New World, that opening further excited and confirmed such expectations, especially on the part of those Renaissance humanists and magi who, in the name and interest of religious revival, promoted the further advancement of science and the useful arts. (Noble 1999, 35)

L'Amérique fut le continent le plus favorable à la promotion de l'idéologie millénariste, parce qu'elle est toujours considérée comme l'incarnation du paradis terrestre, eu égard à son caractère nouveau et vierge. Au $\mathrm{XV}^{\mathrm{e}}$ siècle, Christophe Colomb se déclare comme l'inspiré de Dieu pour trouver une nouvelle route vers l'Inde afin de hâter la réalisation du millenium. Colomb spécifie que "God made me the messenger of the new heaven and the new earth of which he spoke in the Apocalypse of St. John 
after having spoken of it through the mouth of Isaiah» (Sale 1990, 188). C'est en ce sens qu'il considère que la découverte du Nouveau Monde est l'indice concret de l'imminence de la fin du monde et du rétablissement de la perfection perdue. Colomb a identifié cette Nouvelle Terre comme étant le jardin d'Éden et la réalisation de toutes les utopies du monde idéal. Une très vaste littérature américaine reflète cette conviction. On cite à titre d'exemple le livre de John Adolphus Etzler paru en 1833, et intitulé The Paradise Within the Reach of All Men, Without Labor, by Power of Nature and Machinery.

Dans cette lignée millénariste, les posthumanistes s'inspirent du récit de la création pour soutenir le rôle salvateur de la technologie: "L'homme peut, par les arts mécaniques, la retrouver (la perfection perdue) en la domination de la nature qui lui avait été promise dès le sixième jour de la Création» (Lecourt 2003, 74). Les posthumanistes expliquent que Dieu a créé Adam dans un état de perfection à son image et à sa ressemblance et lui a confié la terre pour la dominer et la maîtriser: "Soyez féconds, multipliez, emplissez la terre et soumettez-la; dominez sur les poissons de la mer, les oiseaux du ciel et tous les animaux qui rampent sur la terre» (Gn 1,28). Mais le drame est advenu lorsqu'Adam a voulu manger du fruit de «l'arbre de la connaissance du bien et du mal» en vue de devenir «comme des dieux » $(G n 3,5)$, d'où son péché qui lui a coûté le renvoi du jardin d'Éden (Gn 3,1-24). Toutefois, Adam fut renvoyé du jardin avec une promesse de la part de Dieu qui laisse entrevoir sa victoire finale.

Lorsque la femme s'est justifiée devant Dieu en accusant le serpent de l'avoir séduite, Dieu s'est adressé au serpent en lui disant: "Je mettrai une hostilité entre toi et la femme, entre ton lignage et le sien. Il t'écrasera la tête et tu l'atteindras au talon» (Gn 3,15). Pour les posthumanistes, il n'y a d'autres moyens que la technique pour écraser la tête du serpent et vaincre la faiblesse de l'homme; une technique offerte à l'homme comme le seul moyen pour réaliser la promesse de Dieu et retrouver le chemin du «paradis perdu». Leur conviction peut se résumer ainsi: «Regagner par nos propres forces humaines la perfection d'Adam au Paradis par l'application de notre intelligence à nos moyens techniques» (Lecourt 2003, 75). Ces convictions théologiques millénaristes forment une base commune pour tous les penseurs posthumanistes permettant de parler à leur propos d'une «religion de la technologie» (Noble 1999, 9).

Bref, d'après cette interprétation posthumaniste du texte de la Genèse, le drame de l'humanité semble être divisé en trois temps: un état initial de perfection et de totalité avant le péché; un état d'imperfection et de manque 
suite au péché; puis un processus de salut actuel visant le retour à l'état de perfection initiale. À cet effet, les posthumanistes ne perçoivent dans le manque que son aspect négatif découlant d'une chute de l'humanité, et se dressent comme étant les sauveurs du monde grâce à la puissance de la technologie. Néanmoins, selon une interprétation sémiotique de ce texte des origines, l'enjeu du manque et de la totalité se présente autrement et avec des connotations différentes. À l'encontre de l'approche des posthumanistes qui se fient aux prouesses de la technique pour combattre le manque, une interprétation sémiotique du texte de la Genèse tâche d'ouvrir les horizons et de poser un regard nouveau sur le manque dans sa positivité.

\section{Le manque et la totalité dans le texte de la Genèse}

Prenant conscience du manque qui hante son existence, l'être humain cherche à s'accrocher à tout ce qui paraît en mesure de le sauver de ce soidisant handicap. Pour les posthumanistes, la technologie est sans conteste le meilleur moyen menant à une totalité de l'existence libérée de toute faiblesse, maladie ou mort. Ce recours à la technologie comme seule planche de salut est dû à une conception négative du manque conçu comme une privation et une frustration. Ce que nous proposons dans cet article, c'est une autre vision du manque inspirée d'une lecture sémiotique du texte de la Genèse qui s'inscrit dans une approche d'exégèse synchronique, entrant en dialogue avec la psychanalyse qui «s'intéresse à l'homme comme être de la Parole et de Désir, aux relations que cet être de désir noue» (Beaude 1979, 134).

En analysant la structure discursive du texte de la Genèse, Paul Beauchamp, Louis Panier et Jean Calloud proposent de revisiter certaines affirmations dogmatiques, comme celle du "péché », pour s'ouvrir à une nouvelle interprétation qui s'applique à certaines problématiques du $\mathrm{XxI}^{\mathrm{e}}$ siècle, évoquant essentiellement les concepts de "manque » et de "totalité ». D’après cette lecture, le manque se présente comme étant un élément constitutif de la condition humaine, contrairement à la totalité qui semble être «mortelle». Le manque ne serait plus un vide à combler, mais une réalité à assumer faisant en sorte que «l'homme reconnaît son humanité à partir de ce qui lui manque» (Panier 1996, 89). Cette approche positive du manque qui vient à l'encontre de celle des posthumanistes, permet de lever le voile sur une certaine positivité du manque souvent cachée aux yeux de l'homme contemporain. 


\subsection{De la négativité du manque à sa positivité}

À l'état originel, là où l'être humain est censé être parfait, sans défaut et sans manque, le texte de la Genèse évoque plusieurs états de manque qui caractérisent cette condition originelle: l'être humain n'est pas exempt du travail, il est placé dans le jardin pour le travailler et le garder; il dispose de tous les arbres du jardin, mais avec une interdiction touchant l'arbre de la connaissance; il n'est pas seul, mais il partage son monde avec les animaux et avec son semblable, la femme. D'après ce schéma, il s'avère que le manque est là dès les origines, mais l'être humain ne l'a perçu que dans sa négativité, et le désir du Tout a formé pour lui une aspiration constante le conduisant à vouloir combler son vide existentiel par une possession des «objets» manquants.

Dans le jardin d'Éden, Dieu a créé trois sortes d'arbres: «toute espèce d'arbres séduisants à voir et bons à manger ", «l'arbre de vie » et "l'arbre de la connaissance du bien et du mal»(Gn 2,9). De tous les arbres du jardin, l'homme peut manger, «sauf» de l'arbre de la connaissance. Le «tout» est là, mais tout n'est pas permis. Dieu «interdit à l'homme la totalité comme telle et la déclare mortelle pour lui; et il introduit dans cette totalité une règle de soustraction comme une condition de la vie humaine » (Panier 1996, 85). Pourquoi Dieu le prive-t-il de la totalité ? Où réside le mal dans l'accès à tous les arbres du jardin? Pourquoi l'homme devrait-il supporter et accepter sa condition de manque? Le serpent a tiré avantage de la présence du manque dans le but de détourner la parole de Dieu et ainsi convaincre l'homme de l'aspect négatif du manque.

Le serpent fait croire à l'homme que le manque de la connaissance du bien et du mal le prive de parvenir à la plénitude de son humanité et que cette humanité ne se réalise qu'en devenant «comme des dieux». L'homme a ainsi eu le sentiment d'être écrasé par un Dieu qui a peur que sa créature devienne comme Lui. Ainsi, si Dieu prive l'homme du fruit de l'arbre de la connaissance, c'est pour éviter qu'il ait le pouvoir de la connaissance et le contrôle de la vie. Selon l'insinuation du serpent, le manque imposé par Dieu pourrait être comblé par l'appropriation du fruit de l'arbre interdit; un simple geste de possession sans aucun effort de créativité et de labourage.

Et le résultat? "Ils connurent qu'ils étaient nus»(Gn 3,7). Avant la désobéissance, "tous deux étaient nus, l'homme et sa femme, et ils n'avaient pas honte l'un devant l'autre»(Gn 2,25). Alors qu'après avoir mangé du fruit de l'arbre de la connaissance du bien et du mal, «leurs yeux 
à tous deux s'ouvrirent et ils connurent qu'ils étaient nus; ils cousirent des feuilles de figuier et se firent des pagnes» (Gn 3,7). Ils ont eu honte: "J'ai eu peur parce que je suis $n u$, et je me suis caché» (Gn 3,10). En croyant que la totalité va lui apporter la plénitude de l'existence, l'homme réalise qu'il est toujours en état de manque; ce qui lui cause tant de souffrances. Au lieu de combler le manque, la «prise» du fruit $(\mathrm{Gn} 3,6)$ n'a fait qu'aggraver l'état de manque.

D'ailleurs, le texte de la Genèse suggère une autre approche positive du manque qui dévalorise toute quête acharnée de la totalité. Selon Panier, la totalité équivaut à une fusion avec le monde, sans distinction ni mouvement, alors que la règle de la soustraction permet le mouvement de la vie. Afin de mieux clarifier ce point, revenons aux états de manque du jardin d'Éden pour y voir une positivité.

Dans le jardin des délices, l'espace où Dieu a placé l'homme est un espace marqué par le manque. On le voit par la mention du jardin à "garder» et où Adam doit «travailler». Le jardin n'est pas un garde-manger rempli de victuailles à prendre, mais un espace de travail, de créativité et d'activité. Si le jardin jouissait d'un état de totalité, il n'aurait pas eu besoin d'être travaillé. Il lui manque quelque chose. Mais ce manque est positif puisqu'il incite l'homme à travailler et à être créatif. Le manque n'enlève rien à la valeur de l'homme. Au contraire, il lui donne un rôle primordial dans la gestion du jardin. D'un autre côté, Dieu a créé les animaux pour partager le monde avec l'homme; une manière de montrer à l'homme qu'il ne possède pas seul le jardin, mais qu'il doit le travailler et le garder avec d'autres. À ces animaux de la terre, il manquait un «nom », et c'est Adam qui leur a attribué ce nom. Donner un nom à quelque chose, c'est participer à la création de cette chose et lui assigner un rôle.

Un premier aspect de la condition humaine: le manque fait partie de la nature de l'homme, car celui-ci ne peut pas vivre seul, mais il a toujours besoin de travailler et de partager son monde avec d'autres. Selon cette interprétation du texte de la Genèse, l'homme n'a jamais été créé dans un état de perfection sans manque comme le prétendent les posthumanistes. La spécificité de la perfection humaine résiderait dans son manque, car sans sa présence dans un jardin en état de manque et sans la présence des animaux à ses côtés, l'homme aurait vécu dans l'illusion d'être TOUT tout seul. C'est précisément cette illusion d'être TOUT seul que Dieu voulait épargner à l'homme. Là où «tout est bon » dans l'acte de la création, Dieu voit qu'il n'est pas bon que l'homme soit seul. «En jouant sur les possibilités du français, disons: il n'est pas bon que l'homme soit TOUT... seul» 
(Calloud 1987, 506). Être TOUT seul, c'est vivre dans la solitude de l'amour de soi et exclure l' "autre» de sa vie. C'est ainsi que la création de la femme prend toute son ampleur.

À cet égard, Calloud avertit qu'il ne faut pas être «victimes d'une idée reçue, selon laquelle la création progresse par production de réalités aptes à remplir un vide, selon laquelle ici la femme répondrait au titre d' "objet » au manque diagnostiqué en l'homme" (Calloud 1987, 506). L'aide ne vient pas remplir un vide, mais vise plutôt à libérer l'homme de sa solitude dans le «Tout»: «La femme vient neutraliser le risque de totalité et de complétude. Elle déclare à l'homme son manque et l'accompagne pour qu'il en vive» (Calloud 1987, 506-507). De plus, en jetant «une torpeur sur Adam» (Gn 2,21), Dieu a créé l'" autre» semblable de l'homme dans un état de non-conscience. C'est pour souligner que l'existence de l'autre échappe à la connaissance consciente de l'homme, et qu'il n'est pas tout seul «l'humanité qui est aussi la sienne»(Panier 1996, 89). L'homme partage son humanité avec son semblable et ne détient pas tout seul en tant qu'individu la plénitude de la vie. Le semblable partageant tout avec le semblable n'est donc ni supérieur, ni inférieur. Les deux sont égaux: "La côte perdue est trouvée, non récupérée, elle est devenue trésor, visage d'altérité » (Calloud 1987, 508). Seul et sans le manque, l'être humain n'aurait pas connu sa différenciation en homme (Ish) et femme (Isha): "L'homme est homme d'avoir perdu la côte qu'il trouve construite en femme, il est homme de n'être pas femme; la femme est femme de n'avoir pas été homme. L'un et l'autre existent par ce manque qui les protège d'être complets ou solitaires et d'être complémentaires" (Calloud 1987, 505).

De plus, en précisant que c'est la semence de la femme qui va écraser la tête du serpent, Dieu étale l'humanité à un spectre plus large que celui de l'homme et de la femme. L'humanité des êtres humains ne se limite pas aux individus présents ici et là, mais se déploie dans une descendance à n'en plus finir: "Ni l'homme, ni la femme, ne sont toute l'humanité; elle passe avec la transmission et la génération; elle se diffracte dans les fils... On pourrait dire que la femme porte la douleur de ne pas être à elle seule l'humanité qu'elle transmet» (Panier 1996, 96).

En outre, il vaut d'être remarqué que, dès le début, l'interdit de Dieu a seulement touché l'arbre de la connaissance du bien et du mal, et non l'arbre de vie, parce que cet arbre était «inaccessible» à l'homme. La vie en tant que telle, en tant que totalité, ne peut être contournée par la convoitise de l'homme. Elle n'est pas du même ordre que la connaissance. Elle n'est pas un objet. De plus, en chassant l'homme du jardin des délices pour 
qu'il ne prenne pas de l'arbre de vie, Dieu a voulu lui montrer que la vie échappe à toute emprise et à toute maîtrise. Dieu sait que "le fait d'atteindre et de manger l'arbre de vie constitue un chemin faussé pour acquérir la vie pour toujours» (Thériault 1992, 30). La vie ne s'acquiert pas dans la quête de la totalité. Il lui faut un autre chemin. Couper à l'homme le chemin de l'arbre de vie, c'est lui apprendre que la vie n'est pas une chose maîtrisable. Elle est inaccessible à un acte de possession individuel. La vie est un Tout, mais l'homme ne peut pas être Tout, car «c'est parce qu'il n'est plus tout que le sujet peut naître à tout» (Beauchamp 1990, 123). Etre Tout et se suffire à soi conduit à la mort, tandis qu'assumer son manque, c'est s'ouvrir à Tout et à la Vie. D'après cette approche positive du manque, l'état de perfection initiale de l'homme s'avère un état de manque où l'harmonie et la créativité sont continuellement à l'œuvre. C'est l'état de manque qui pousse constamment l'homme à chercher et à désirer l'autre dans sa vie, et à réaliser la plénitude de sa condition humaine dans cette présence active et vivifiante.

\subsection{La condition humaine entre deux espaces}

Selon cette analyse du texte de la Genèse, il semble que le manque et la totalité constituent deux espaces caractérisant la condition humaine. En lui posant la question «où es-tu ?» (Gn 3,9), Dieu demande à Adam dans quel espace il se situe. Dans l'espace de la condition humaine donnée par Dieu à l'homme, mais marquée par le manque positif, ou bien dans l'espace voulu par l'homme visant la totalité «mortelle»? En réponse aux posthumanistes qui ont fait leur choix de bien vouloir combler le vide du manque par un passage à l'espace de la totalité mortelle, le texte de la Genèse propose une autre approche de l'espace de la totalité basée sur une vision positive du manque.

La condition initiale de l'homme semble être inscrite dans l'espace où la «totalité » est soustraite à l'homme: «Dans l'interdit inaugural $(2,16)$, le schéma "Tout sauf" détermine que ce n'est pas une partie du tout, mais la totalité en tant que totalité qui est interdite dès le point de départ de l'itinéraire langagier " (Beauchamp 1990, 122). D'ailleurs, l'avertissement de Dieu est clair: «Le jour où vous en mangerez, vous mourrez de $\operatorname{mort}^{7}$ » (Gn 2,17). Le jour où vous prendrez du fruit de l'arbre de la connaissance du bien et du mal, le jour où vous quitterez l'espace de votre condition

7. Traduction française de la LXX. 
humaine limitée et vous aspirerez à la totalité, vous mourrez. Pourquoi, et de quelle mort parle-t-il?

En empiétant sur l'espace de la totalité, l'homme a réalisé qu'il était nu. Il a pris conscience de sa mortalité, et il a eu peur. Pourtant, la nudité avait été d'emblée présente, mais elle ne suscitait pas la honte. Le manque était toujours là, mais il était comblé par une présence de Dieu qui communique son amour avec l'homme à travers la parole. Le serpent fait irruption dans cet espace de relation harmonieuse entre Dieu et l'homme en détournant la parole de Dieu et en la réduisant en un savoir malfaisant. La relation est rompue entre l'homme et Dieu par la déviation de la parole de Dieu et par le soupçon quant à l'intention de cette parole. À la place de «l'entendre», le serpent propose «le voir».

Dans le contexte de la condition originelle de l'homme "sans honte", la parole était à l'œuvre: "Yahvé Dieu fit à l'homme ce commandement" (Gn 2,16); "Yahvé Dieu dit» (Gn 2,18); "celui-ci s'écria» (Gn 2,23). La relation entre Dieu et l'homme était basée sur la parole, cette parole créatrice par laquelle tout a été fait. En voulant pervertir cette relation de confiance, le serpent s'adresse à la femme en disant: «Dieu sait que, le jour où vous en mangerez, vos yeux s'ouvriront et vous serez comme des dieux, qui connaissent le bien et le mal» (Gn 3,5). Il lui propose d'opter pour le «voir» à la place de l' "entendre». D'ailleurs, dans la Bible, l'enjeu principal de la relation de Dieu à l'homme repose sur l'injonction: "Écoute Israël ». À chaque fois que Dieu s'adresse à son peuple, il exige l'écoute, alors que le peuple veut «voir» et «savoir»: «Je vais faire un détour pour voir...» $(\operatorname{Ex} 3,3)$; «Tu ne peux pas voir ma face, car l'homme ne peut me voir et vivre" (Ex 33,20). Le peuple dit à Aaron: "Allons, fais-nous un dieu qui aille devant nous, car ce Moïse, l'homme qui nous a fait monter du pays d'Égypte, nous ne savons pas ce qui lui est arrivé » (Ex 32,1). Selon le discours du serpent, il ne s'agit plus d'une parole à partager comme don entre Dieu et l'homme, mais d'un «voir» et d'un «savoir». Ce que le serpent propose, c'est un "savoir» scientifique de Dieu qui est de l'ordre $\mathrm{du}$ «voir» et qui vient remplacer l'«entendre». Il faut voir pour savoir. Entendre n'est pas suffisant pour répondre au désir de l'homme. Il faut prendre et manger pour que les yeux s'ouvrent et que l'homme connaisse le bien et le mal.

Pourtant, le résultat n'est pas conforme à la promesse du serpent. Avant de posséder la connaissance de Dieu et du monde, l'homme a commencé par se connaître. La transformation dans le voir s'est produite à l'intérieur de l'homme, sur le plan de sa relation à lui-même: "Ils connurent 
qu'ils étaient nus»(Gn 3,7). Oui, le voir a conduit à un savoir et à un jugement: celui de la nudité de l'homme et de son état de manque.

Si l'homme veut voir, c'est qu'il lui manque quelque chose à la vue; il est conscient de son état de manque et il veut combler ce manque par le «savoir» du «voir». Or, dans le «voir », l'objet reste extérieur à la personne et suscite continuellement un désir de possession. Alors que dans l' "entendre », tout se passe à l'intérieur de la personne et nécessite de sa part une transformation. À cause d'une déficience dans l'entendre, l'homme n'a réalisé dans le manque de sa condition humaine qu'une privation. S'il avait consenti à une relation interpersonnelle telle qu'établie par Dieu, basée sur la parole, sur la communication, sur l'entendre, sur l'écoute et sur l'Alliance, il aurait su que le manque de sa condition est plutôt positif, car il le pousse continuellement à puiser son mystère et celui de l'autre dans l'émerveillement. À travers la communication de la parole, l'homme vit dans un état continuel de découverte de soi-même et de l'autre. Il écoute l'autre et exige de même l'écoute de l'autre. Il découvre l'autre et se découvre lui-même à travers l'autre. Or, le savoir vient rompre cette relation à soi et à l'autre. Ce n'est pas le savoir en soi, mais le savoir-pouvoir-jugement qui est en cause ici.

En accédant au «savoir ", l'homme acquiert par le fait même un "pouvoir» sur les choses. Et une fois détenteur du pouvoir, l'homme se place comme un juge pour arbitrer l'autre en calculant le bien et le mal. Dieu voulait garder ce privilège pour lui, parce que juger l'autre n'est pas une simple affaire de connaître le bien et le mal, mais une connaissance vraie et profonde de l'autre. C'est une reconnaissance de la valeur de l'autre audelà du bien et du mal, car c'est Dieu seul qui «sonde les cœurs et les reins» (Ps 7,10). Juger l'autre à partir d'une équation dont les seuls paramètres sont le bien et le mal, c'est réduire l'être humain à une machine d'exécution du bien et du mal et d'oppression de l'autre à dominer.

De la sorte, réduire l'espace de la vie humaine au seul espace de la totalité, c'est réduire la connaissance de soi et de l'autre au seul savoir scientifique, au pouvoir et au jugement. Opter pour l'espace de la totalité à la manière posthumaniste, c'est limiter l'existence humaine à une simple équation mathématique encadrant l'homme dans des paradigmes et des définitions. C'est changer les conditions de la vie humaine et s'embarquer dans l'espace de la mort. 


\section{Conclusion}

À la lumière de notre lecture du texte de la Genèse, le posthumain se présente comme étant l'Adam qui refuse sa condition humaine, et se place dans l'espace du "Tout». Ce qui lui manque à la possession du "Tout», c'est la maîtrise du savoir technique permettant de transformer et d'améliorer la condition biologique de l'être humain. La perfection humaine et la totalité de l'existence seraient ainsi réduites à l'obtention d'une nouvelle condition post-humaine et post-biologique. Ceci explique pourquoi les posthumanistes croient fermement que l'angoisse existentielle des êtres humains se dissipe dans ce passage à la condition post-humaine qui prétend combler le manque de la condition humaine en triomphant sur la mort.

Il s'avère que cette approche posthumaniste du manque est bien accueillie dans un terrain d'attente caractéristique du contexte social de consommation. Dans nos sociétés occidentales contemporaines, le bonheur est construit socialement autour de la consommation: plus on consomme et plus on possède des objets, plus on devrait être heureux. Le manque de notre condition humaine causant notre angoisse existentielle pourrait être ainsi comblé par des objets de consommation. Dans ce contexte de consommation, la mort qui poussait jadis l'homme à se questionner sur sa vie et sur l'après de sa vie n'est devenue qu'un «objet» à vaincre. À cet effet, les posthumanistes ne seraient que l'épiphénomène de cette négation essentielle du manque et de la mort. C'est dans cet espace de normativité sociale que les promesses du posthumanisme gagnent du terrain auprès de beaucoup de gens sans qu'ils soient forcément posthumanistes.

Cette acceptation sociale empêche souvent de remarquer qu'en voulant fuir le manque et posséder le Tout, le posthumain ne fait que sombrer dans un état de mort. S'il manque quelque chose, c'est qu'il faut faire quelque chose. Et quand il ne manque rien, il ne reste plus rien à faire. Un être saturé et possédant le «Tout » n'a plus rien à espérer: c'est la mort. À cet égard, vouloir retourner à un prétendu état de perfection initiale ne s'avère qu'une illusion mortelle. L'état initial de l'Adam était sûrement serein, mais non parfait, car la perfection ne s'appréhende jamais comme un état statique de plénitude où l'homme ne manque de rien.

Posthumain, où es-tu? Dans l'espace de la condition humaine marquée par le manque, ou bien dans l'espace d'une condition posthumaine parfaite libérée de «tout» manque?

Humain, où es-tu? Écrasé par le manque de ta condition humaine ou bien ébloui par les promesses de la condition posthumaine? 
Entre manque et totalité, où se situe la condition humaine? Faut-il cristalliser la condition humaine dans un état de manque et ainsi diaboliser tout progrès technique visant la perfection? Faut-il, au contraire, chercher à tout prix la réalisation de la perfection humaine en éradiquant tout manque dans une potentielle condition post-humaine?

Une piste de réponse pourrait être inspirée du texte de la Genèse: si le "Tout» est mortel pour l'homme, il n'est pas bon en revanche que l'homme soit seul dans un état de manque. En conséquence, ni le manque, ni la totalité ne semble être exclusivement caractéristique de la condition humaine. Le manque dans son état stationnaire n'est pas une valeur en soi. De même, la totalité dans son aspect statique ne fait qu'écraser l'homme et l'étouffer. Ainsi, restreindre le choix de l'homme à ces deux états extrêmes, c'est figer la condition humaine dans un état statique de mort, et c'est éliminer la possibilité de l'existence d'un entre-deux. Ce que le texte de la Genèse propose, c'est un mouvement continuel et positif allant de l'état de manque vers l'état de la totalité et vice versa. Le couple du jardin d'Éden a goûté à la Vie dans cet espace de l'entre-deux, dans ce va-et-vient qui ne tourne pas en cercle vicieux, mais en cercle herméneutique. Le manque n'est que cet espace qui permet à l'autre d'exister et de participer au cheminement vers la totalité du vivre ensemble.

$\mathrm{Au}$ lieu de proposer, à la manière des posthumanistes, la totalité comme une solution unique pour déployer la condition humaine, il serait possible de s'interroger sur le mouvement vital d'une condition humaine qui échappe à la fixation du manque et de la totalité.

\section{Références}

Beauchamp, P. (1990), L'un et l'autre Testament 2. Accomplir les Écritures, Paris, Seuil (Parole de Dieu).

Beaude, P.-M., (1979), Tendances nouvelles de l'exégèse, Paris, Le Centurion (Tendances nouvelles).

Bostrom, N. (2000) [anglais 1998], "Qu'est-ce que le transhumanisme? ", <http://www.transhumanism.org/index.php/WTA/more/147/>.

Calloud, J. (1987), «Pour une analyse sémiotique de la Genèse 1 à 3 ", dans La création dans l'Orient Ancien, Paris, Cerf (Lectio Divina 127), p. 483-513.

Kurzweil R. (1999), The Age of Spiritual Machines. When Computers Exceed Human Intelligence, New York, Viking. 
La Bible de Jérusalem (1975), Paris, Desclée De Brouwer.

Lecourt, D. (2003), Humain, posthumain, Paris, PUF (Science, histoire et société).

Moravec, H. (1988), Mind Children. The Future of Robot and Human Intelligence, London, Harvard University Press.

Noble, D. (1999), The Religion of Technology. The Divinity of Man and the Spirit of Invention, New York, Penguin Books.

Panier, L. (1996), Le péché originel. Naissance de l'homme sauvé, Paris, Cerf (Théologies).

Sale, K. (1990), The Conquest of Paradise. Christopher Columbus and the Columbian Legacy, New York, Knopf.

Thériault, J.-Y. (1992), «L'Adam dans le jardin, deuxième partie », Sémiotique et Bible, 68, p. 15-34.

Tipler, F. (1994), The Physics of Immortality. Modern Cosmology, God and the Resurrection of the Dead, New York, Doubleday.

\section{Résumé}

Le «manque » et la «totalité » sont deux traits caractéristiques de la condition humaine dans sa quête de soi depuis les mythes fondateurs jusqu'au progrès phénoménal de la technologie au $\mathrm{XXI}^{\mathrm{e}}$ siècle. Néanmoins, ces deux concepts revêtent des connotations différentes et souvent contradictoires selon le contexte et les aspirations qui les sous-tendent. En quête de l'humanité parfaite, le posthumanisme prône la totalité comme le but ultime de la vie humaine, et refuse catégoriquement le manque dans son aspect négatif et écrasant. Par contre, une lecture sémiotique du texte de la Genèse révèle que le manque fait partie intégrante de la condition de l'homme, en opposition à la totalité qui s'avère mortelle. À cet égard, comment une nouvelle interprétation d'un texte datant de milliers d'années a le potentiel de jeter un regard nouveau sur des questions d'actualité? Comment se déploient les espaces de la condition humaine entre manque et totalité ?

\section{Abstract}

"Lack» and "totality» are two features of the human condition in its quest for self from founder myths to phenomenal progress of technology in the twenty-first century. Nevertheless, these two concepts are of different 
connotations and often contradictory depending on the context and aspirations that underlie them. In search of the perfect humanity, posthumanism advocates the "totality" as the ultimate goal of human life, and categorically refuses the "lack» because of its negative and overwhelming aspect. On the other hand, a semiotic reading of the text of Genesis reveals that "lack» is not but an integral part of the human condition, as opposed to "totality" that is lethal. In this regard, in which manner a new interpretation of a text dating back thousands of years has the potential to shed new light on current issues? How to deploy the spaces of the human condition between «lack» and «totality»? 